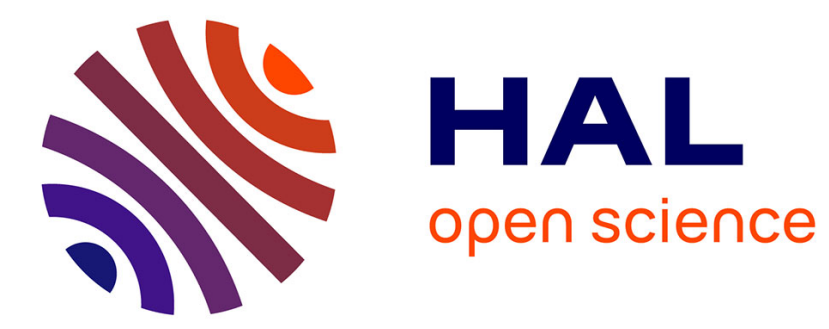

\title{
Sémiotique et anatomie chez Leibniz et Diderot
}

Claire Fauvergue

\section{To cite this version:}

Claire Fauvergue. Sémiotique et anatomie chez Leibniz et Diderot. Dix-Huitième Siècle, 2005, 37 (1), pp.483-496. 10.3406/dhs.2005.2688 . hal-03241547

\section{HAL Id: hal-03241547 \\ https://hal.science/hal-03241547}

Submitted on 28 May 2021

HAL is a multi-disciplinary open access archive for the deposit and dissemination of scientific research documents, whether they are published or not. The documents may come from teaching and research institutions in France or abroad, or from public or private research centers.
L'archive ouverte pluridisciplinaire HAL, est destinée au dépôt et à la diffusion de documents scientifiques de niveau recherche, publiés ou non, émanant des établissements d'enseignement et de recherche français ou étrangers, des laboratoires publics ou privés. 


\section{Sémiotique et anatomie chez Leibniz et Diderot}

\section{Claire Fauvergue}

\section{Citer ce document / Cite this document :}

Fauvergue Claire. Sémiotique et anatomie chez Leibniz et Diderot. In: Dix-huitième Siècle, $n^{\circ} 37,2005$. Politiques et cultures des Lumières. pp. 483-496;

doi : https://doi.org/10.3406/dhs.2005.2688

https://www.persee.fr/doc/dhs_0070-6760_2005_num_37_1_2688

Fichier pdf généré le 17/05/2018 


\section{SÉMÉIOTIQUE ET «ANATOMIE » ${ }^{1}$ CHEZ LEIBNIZ ET DIDEROT}

Y. Belaval parle à l'endroit de Leibniz de «philosophie de l'invention ". Il se réfère pour cela à la Dissertatio de Arte Combinatoria $^{2}$ rédigée en 1666 . L'invention serait pour Leibniz « combinatoire » ou « opératoire » et se distinguerait d'une « théorie, qui se nommerait assez bien vitaliste, de l'invention ». Y. Belaval pense ici à Diderot et émet l'idée selon laquelle « l'invention de l'intellect combinatoire et l'invention de l'enthousiasme ont une racine commune $\gg{ }^{3}$. Or la perception n'est-elle pas déjà par elle-même combinatoire ou opératoire, ce qui permet justement à Y. Belaval de formuler l'hypothèse d'une "racine commune »? Si l'hypothèse est juste, il y aurait quelque similitude entre l'invention qui procède par une combinatoire intellectuelle et l'invention qui procède par une sorte de combinatoire sensible et résulte moins de l'exercice de la raison que de celui de la mémoire ou de l'imagination.

Cette «racine commune » relève de la perception. Leibniz conçoit, dès ses écrits de jeunesse et son de Arte Combinatoria auquel se réfère $\mathrm{Y}$. Belaval, un « fil d'Ariane » ${ }^{4}$. Il entend par cette métaphore un «procédé sensible et mécanique » ${ }^{5}$ pour conduire la pensée et plus exactement un «fil palpable », selon

1. Le terme d' « anatomie » est employé par Leibniz et par Diderot. Si « anatomie » est un terme médical, il a également le sens figuré d' «analyse ", et c'est ainsi que Leibniz l'emploie. Quant à Diderot, il est probable qu'il joue sur la polysémie du terme lorsqu'il fait l'esquisse d'une «anatomie métaphysique». Leibniz, Réponse aux réflexions contenues dans la seconde Édition du Dictionnaire Critique de M. Bayle, article Rorarius, sur le Système de l'Harmonic préétablie, GP IV, 562, et Diderot, Lettre sur les sourds et muets, DPV IV, 140.

2. Leibniz, Dissertatio de Arte Combinatoria, GP IV, 27-104. Voir H. Ishiguro, Leibniz's philosophy of logic and language, (London, 1972, rééd. Cambridge University Press, 1990).

3. Y. Belaval, Études leibniziennes de Leibniz à Hegel, (Paris, Gallimard, 1976), pp. 387-389.

4. La métaphore du «fil dans le labyrinthe » apparaît en outre dans les écrits français. Leibniz, Préceptes pour avancer les sciences, GP VII, 157.

5. L. Couturat, La logique de Leibniz, 1901, rééd. Olms, (1969), p. 90. 
une terminologie qui accorde un rôle non négligeable au toucher. Leibniz emploie par ailleurs les métaphores de " criterium palpable de la vérité " ainsi que de "démonstrations palpables " 6 lorsqu'il formule le projet d'une science générale, comme le relève L. Couturat. Rappelons que Diderot définit pour sa part l'analogie, dont procède le jugement, comme une «espèce de fil qui n'est pas entre les mains de tout le monde ${ }^{7}{ }^{7}$. La proximité avec certains développements de la Lettre sur les aveugles concernant le rapport entre toucher et abstraction nous amène à envisager le rôle du sensible pour la séméiotique conçue par les deux auteurs.

Leibniz considère qu'il nous est impossible d'affirmer «que ce qui nous paraît est une réalité ». Il explique que l' " on ne peut rien conclure sur la réalité des choses. Toutes nos expériences montrent simplement qu'il y a une liaison dans nos apparences $"{ }^{8}$. Leibniz reproche par ailleurs aux sceptiques d'avoir cherché « une plus grande réalité dans les choses sensibles hors de nous que celle de phénomènes réglés ${ }^{9}$. Autrement dit, le sensible n'est qu'un certain ordre de phénomènes. Car, si l'ordre est préétabli, il n'est pas immédiatement perceptible. Leibniz explique que vouloir lire dès à présent l'« ouvrage de l'univers », qu'il compare à un «grand et vrai poème», c'est comme «vouloir prendre le roman par la queue et prétendre d'en déchiffrer l'intrigue dès le premier livre, au lieu que la beauté d'un roman est d'autant plus grande qu'il sort plus d'ordre enfin d'une plus grande confusion apparente ». Pour rétablir l'ordre du poème du monde, ou encore pour que l'ordre de nos phénomènes suive celui des êtres hors de nous, il faut vérifier expérimentalement leur liaison par l'exercice patient des sens. Ce qui signifie que le phénomène ne peut être produit par la sensation isolée. Les liaisons des phénomènes

6. Leibniz, Initia et Specimina Scientiae generalis, GP VII, pp. 57, 59 et 125. cité dans L. Couturat, ouvr. cité, p. 91.

7. Diderot, Encyclopédie, article ENCYCLOPÉDIE, DPV VII, 222.

8. Leibniz, Correspondance Leibniz-Foucher, GP I, 372. Voir sur ce point, Frédéric de Buzon, «L'harmonie : métaphysique et phénoménalité », Revue dé Métaphysique et de morale, numéro 1, 1995, pp.95-120. L'auteur souligne la portée phénoménale de l'hypothèse de l'harmonie prétablie et cite Leibniz: « je n'ai tâché de rendre raison que des Phénomènes, c'est-à-dire du rapport dont on s'aperçoit entre l'Ame et le corps ». Remarques de l'auteur du système de l'harmonie préétablie, Journal de Trévoux, janvier 1708, GP VI, 595.

9. Leibniz, Éclaircissement des difficultés que $M$. Bayle a trouvées dans lé système nouveau de l'union de l'âme et du corps, GP IV, 523. Le rapport entre le scepticisme et le phénomène a été étudié par Jean-Paul Dumont, Le Scepticisme et le phénomène, (Paris, Vrin, 1985). 
résultent de sensations dont il faut recomposer la suite par une multitude d'opérations pour la plupart inconscientes. Ainsi, l'expérience permet non pas d'affirmer mais d'évaluer la conformité entre la perception et la réalité phénoménale. Cette conformité se vérifie, selon Leibniz, par la capacité de « reconnaître et d'inventer de l'ordre et de l'artifice $\gg 10$. Elle se vérifie encore par l'aptitude que nous avons à «prédire avec succès des apparences futures " 11 . Dès lors, "la théorie peut prévenir la pratique " 12 , l'invention précéder la découverte ${ }^{13}$ et le jugement prévenir l'expérience ${ }^{14}$. L'ensemble de ces remarques confirme l'hypothèse selon laquelle le réel, tout en étant préétabli, ne se laisse saisir que par un ordre qu'il faut inventer; une invention et un artifice qui obéissent à certaines lois.

Diderot, de son côté, explique que la sensation présente, immédiate et isolée, n'aurait aucune réalité pour nous si elle n'était liée à la suite des perceptions conservées par la mémoire : « qu'est-ce que la voix du présent ? Rien », répond Diderot, pour lequel le «présent n'est qu'un point et la voix que nous entendons est toujours celle de l'avenir ou du passé ${ }^{15}$. La perception rétablit ainsi une continuité qui redouble celle de la réalité mais n'obéit pas aux mêmes lois : les lois qui régissent la composition de suites de sensations ne sont pas celles qui régissent l'enchaînement des êtres et des phénomènes. L'ordre que nous percevons est notre propre ouvrage. Diderot, conscient du problème insoluble légué par le scepticisme, comme des apories de l'immatérialisme de Berkeley, s'exclame : «Que dirais-je à celui qui prétendant que quoi qu'il voit, quoi qu'il touche, quoi qu'il entende, qu'il aperçoive, ce n'est pourtant jamais que sa sensation qu'il aperçoit : qu'il pourrait avoir été organisé de manière que tout se passât en lui, comme il s'y passe, sans qu'il n'y ait rien au dehors (...) ? $\gg 16$.

10. Leibniz, Méditation sur la notion commune de justice, dans Leibniz, Le droit de la raison, (Paris, Vrin, 1994), p. 118. et pp. 116-117.

11. Leibniz, Correspondance Leibniz-Foucher, GP I, 372.

12. Leibniz, Préceptes, GP VII, 172.

13. Leibniz, À Des Maizeaux, GP VII, 536. «Démocrite a prévu les étoiles insensibles dans la voie lactée avant la découverte des télescopes ».

14. Leibniz, Préceptes, GP VII, 171.

15. Diderot, Lettres sur la postérité, DPV XV, 30.

16. Diderot, Encyclopédie, article PYRPHONIENNE ou SCEPTIQUE (PHILOSO$P H I E$ ), DPV VIII, 160. Voir le commentaire de l'article par J. P. Dumont, ouvr. cité., pp. 64-66. 
Si l'on se réfère aux analyses développées dans la Lettre sur les aveugles, ce n'est pas la sensation en elle-même mais l'ordre que nous lui imprimons qui peut effectivement être considéré comme notre propre ouvrage : il résulte d'une combinatoire inconsciente. Diderot revient sur cette idée dans les Lettres sur la postérité, où il compare le pressentiment de la postérité à la sensation présente et prend l'exemple d'un concert lointain. Les sons de ce concert lointain sont épars et imperceptibles, mais la sensation est capable d'en faire un chant suivi. À celui qui demande si ce concert est bien réel, Diderot répond : «Quel concert plus réel que celui que j'entends, et dont je suis en état de chanter toute la mélodie et tous les accompagnements ? ». Il en est de même du phénomène. Il résulte d'une sorte d'analogie entre la sensation et la réalité, analogie qui apparaît dès que la sensation est liée. Mais une fois liée, cellc-ci ne coïncide jamais parfaitement avec l'instant présent. Diderot estime en effet que l' « idée du présent et celle de l'avenir sont inséparables ». L'analogie sensible entre les sensations présentes et futures nous destine à exister par anticipation : «Demain n'est pas plus pour vous que l'année 99999 », mais « le ton est donné et il ne changera pas ${ }^{17}$. L'anticipation du réel et de son ordre résulte d'une combinatoire sensible.

Les concepts d'ordre et de réel ${ }^{18}$ mettent en jeu la question des signes. La séméiotique est tout d'abord conçue dans le cadre du projet encyclopédique dont Leibniz est l'un des initiateurs. Car elle est liée au problème de la division des sciences. Le concept de sêmeiotikê est connu pour avoir été introduit par Locke qui conçoit une division « encyclopédique » des sciences et distingue la Physique, la Morale et la Logique ou science des signes. Celle-ci, encore dénommée « séméiotique » ${ }^{19}$, a pour objet les idées et les mots. Or Leibniz, interlocuteur de Locke dans les Nouveaux Essais sur l'entendement humain, voit là une difficulté. Il distingue pour sa part «la connaissance des étymologies des mots et des usages des langues » et la «science de raisonner, de juger, d'inventer " ${ }^{20}$. Si l'on parle de séméiotique

17. Diderot, Lettres sur la postérité, DPX XV, pp. 30 et 44.

18. «Le mot encyclopédie signifie enchaînement des sciences 》. Diderot, Encyclopédie, Prospectus, DPV V, 85.

19. J. Locke, Essai sur l'entendement humain, livre IV, chapitre XXI, paragraphe 4. La séméiotique constitue d’abord une extension de la logique.

20. Leibniz, Nouveaux essais sur l'entendement humain, GP V. 504. 
dans les deux cas, il s'agit en fait de deux sciences distinctes: l'une concerne les mots, l'autre les idées, et il y a tout lieu de croire qu'elles n'obéissent pas à la même logique. Car l'ordre des connaissances n'est pas celui des choses : « la plupart des choses [peuvent] être regardées de plusieurs faces », explique Leibniz. Il en est de même de «l'ordre des systèmes ", si l'on considère qu' «il y a ordinairement autant de sentiments que de têtes ${ }^{21}$. La séméiotique, indissociable de l'ordre encyclopédique qu'elle fonde, n'en est pas une division à proprement parler. L'enchaînement des connaissances ${ }^{22}$ ainsi que la division des sciences devraient suivre les principes de la séméiotique, entendue comme « art d'inventer » ${ }^{23}$. L'Encyclopédie est conçue par Leibniz comme une combinatoire ${ }^{24}$.

Ainsi les diverses sciences distinguées par Locke sont tout au plus pour Leibniz des «arrangements divers des mêmes vérités » ${ }^{25}$. L'ordre alphabétique ou systématique n'a qu'une valeur provisoire et son défaut d'ordre logique doit être compensé par l'introduction d'un système de « renvois » ${ }^{26}$. La distinction d'une conception linguistique et d'une conception «pré-linguistique » de la séméiotique rejoint celle de la « langue universelle » et de la «Caractéristique universelle ». Celle-ci, définie plus particulièrement par Leibniz dans sa Méthode de l'Universalité, peut être considérée comme une science générale des signes: elle met « des caractères à la place des choses » et dépasse largement l'étude linguistique proprement dite, car «c'est la Caractéristique

21. Leibniz, Discours touchant la méthode de la certitude et l'art d'inventer, GP VII, 180.

22. Leibniz, Correspondance Leibniz-Foucher, GP I, 370. Leibniz remarque qu'il arrive que «l'ordre de notre connaissance» diffère de l'«ordre de la nature ».

23. Leibniz, Discours, GP VII, 174-183. Leibniz déclare qu'il est « obligé quelques fois de comparer nos connaissances à une grande boutique ou magasin ou comptoir sans ordre et sans inventaire ». Le projet encyclopédique leibnizien intéresse autant la pratique que la théorie et s'étend aux sciences et aux arts. Il se présente comme l' "Inventaire Général de toutes les connaissances qui se trouvent déjà parmi les hommes ». GP VII, 182.

24. Y. Belaval, Études leibniziennes de Leibniz à Hegel, ouvr. cité, pp. 43 et 45 .

25. Leibniz, Nouveaux essais, GP V, 505-506. Une «même vérité peut-être placée en différents endroits » de l'ordre des connaissances. Leibniz compare l' «Inventaire Général » des connaissances à l'inventaire d'une bibliothèque et remarque que «ceux qui rangent une bibliothèque ne savent bien souvent où placer quelques livres, étant suspendus entre deux ou trois endroits également convenables ».

26. Leibniz, Discours, GP VII, 178. 
qui donne les paroles aux langues, les lettres aux paroles, les chiffres à l'Arithmétique, les notes à la Musique ${ }^{27}$. Elle définit un ordre véritablement «scientifique » ${ }^{28}$, l'ordre des vérités et non celui des mots ; d'où le projet leibnizien d' « une Encyclopédie démonstrative », qui obéisse à une "vraie Logique " ${ }^{29}$ et où l'on pourrait «tout trouver par le secours de la science générale ou de l'Art d'inventer $\gg{ }^{30}$. Or l'ordre des vérités se distingue autant de l'ordre « naturel » que de l'ordre dit « accidentel » des idées. Lorsqu'il passe de la séméiotique à l'«anatomie de la pensée » ${ }^{31}$, Leibniz distingue en effet l' « ordre naturel des idées » et l'ordre «que les occasions ou les accidents où notre espèce est sujette nous a fourni », dans lequel entre «du hasard et de l'accidentel ». Cet ordre accidentel n'est ni naturel ni artificiel, dans la mesure où les règles n'en sont pas fixées. Il forme tout au plus une histoire, mais nc constitue pas une science. L' « histoire de nos découvertes ${ }^{32}$ nous en fournit un bon exemple. Quant à l'ordre des vérités qui intéresse le projet encyclopédique leibnizien, il relève de la séméiotique et non de l'histoire. Selon les termes de la séméiotique ainsi redéfinie, la production de l'ordre suit le processus suivant : «plus on découvre de vérités et plus on est en état d'en remarquer une suite réglée et de se faire des propositions toujours plus universelles ». Il en résulte une abréviation des connaissances et des sciences similaire à celle que l'on rencontre dans les mathématiques ; d'où le « paradoxe » selon lequel « les sciences s'abrègent en s'augmentant ${ }^{33}$, paradoxe qui rend compte de l'encyclopédisme de Leibniz et de Diderot.

27. Leibniz, De la Méthode de l'Universalité. (1674), paragraphe 4, cité et commenté par L. Couturat, ouvr. cité, p. 90.

28. Leibni\%, Discours, GP VII, 180.

29. Leibniz, Préceptes, GP VII, pp. 168 et 172.

30. Leibniz, Discours, GP VII, 180. Leibniz considère qu' « il y a des secrets dans l'art de penser, comme dans les autres Arts, et que c'est là l'objet de la Science générale ». GP VII, 183.

31. Leibniz. Réponse aux réflexions contenues dans la seconde Édition du Dictionnaire Critique de M. Bayle, article Rorarius, sur le Sy'stème de l'Harmonie préétablie, GP IV, 562.

32. Leibniz, Nouveaux essais, GP V, 255-256. Condillac reprendra la distinction envisagée par Leibni\%. lorsqu’il propose une division des signes en « signes accidentels », "signes naturels » et "signes d'institution ». Condillac, Essai sur l'origine des connaissances humaines, $I^{\mathrm{C}}$ partic, II, IV, paragraphe 35.

33. Leibniz, Discours, GP VII, 180. Leibniz déclare que " plus une science est perfectionnée, et moins a-t-elle besoin de volumes ». Diderot énonce une idée similaire dans l'Encyclopédie et oppose l'encyclopédisme à l'académisme. Diderot, Encrelopédie, article ENCYCIOPYIDI:, DPV VII, 227. 
Nous envisagerons dans ce qui suit la « séméiotique des encyclopédistes ${ }^{34}$ et plus précisément celle de Diderot. Celui-ci remarque dans l'article ENCYCLOPÉDIE dont il est l'auteur qu' "après avoir traité la science des signes et des sons avec assez de légèreté, il y a eu un temps où de bons esprits reconnurent qu'elle avait avec la science des choses plus de liaison qu'ils n'en avaient d'abord soupçonné, et qu'on pouvait regarder cette spéculation comme n'étant point du tout indigne de la philosophie » ${ }^{35}$. Diderot écrit encore dans l'article LOCKE : «Malgré tout ce que Locke et d'autres ont écrit sur les idées et sur les signes de nos idées, je crois la matière toute nouvelle " ${ }^{36}$. Diderot aborde la question de la « science des signes ${ }^{37}$ dans le Prospectus de l'Encyclopédie. Il distingue d'une part les «signes de la parole » ${ }^{38}$, ce sont les « sons articulés », et d'autre part les caractères de l'écriture et les gestes. Il envisage aussi les « signes de la pensée » ${ }^{39}$ et distingue la « science des signes » et la «science des choses ». Enfin, il conçoit une "science générale » ou une «métaphysique des choses » ${ }^{40}$, dont dépend l'explication des phénomènes et dont procèdent les différents arts et sciences ${ }^{41}$.

Toujours dans le Prospectus de l'Encyclopédie, le terme de « séméiotique » ${ }^{42}$ désigne une des divisions de la médecine, une pratique médicale qui donne lieu à un article non signé intitulé SÉMÉIOTIQUE, ou SÉMÉIOLOGIE ${ }^{43}$. La science médicale des

34. Le sujet a été étudié par Sylvain Auroux, La séméiotique des encyclopédistes, (Paris, Payot, 1979). L'auteur étudie l'article ENCYCLOPÉDIE de Diderot en le confrontant à la linguistique des Cours de Saussure, pp. 74-76.

35. Diderot, Encyclopédie, article ENCYCLOPÉDIE, DPV VII, 191.

36. Diderot, Encyclopédie, article LOCKE PHILOSOPHIE DE, DPV VII, 713.

37. Diderot, Encyclopédie, Prospectus, DPV V, 110. Les caractères de l'écriture peuvent être « idéaux », « hiéroglyphiques », « héraldiques » ou «alphabétiques ».

38. Diderot, Encyclopédie, article ART, DPV V, 495.

39. Diderot, Encyclopédie, Prospectus, DPV V, 110.

40. Diderot, Encyclopédie, article ENCYCLOPÉDIE, DPV VII, pp. 210 et 220. R.L. Amstrong qualifie la séméiotique lockienne de "nouvelle métaphysique », une remarque qui pourrait s'appliquer à Diderot. R.L. Amstrong, «John Locke's doctrine of signs : a new metaphysics », Journal of the history of ideas, XXVI, (1965), pp. 369-382.

41. Leibniz écrit de même qu' « en examinant chaque science, il faut tâcher d'en découvrir les principes d'invention ». Préceptes, GP VII, 168.

42. Diderot, Encyclopédie, Prospectus, DPV V, 115. Diderot se réfère à Boerhaave qui divise la science médicale en physiologie, pathologie, séméiotique et thérapeutique.

43. Encyclopédie, article SÉMÉIOTIQUE ou SÉMÉIOLOGIE, (Médecine séméiotique), non signé, Enc. XIV, 937 a. 
signes y est abordée à partir des symptômes et des phénomènes en général. Diderot n'en est pas l'auteur, mais développe à notre avis une conception «existentielle » des signes qui semble largement inspirée de la séméiotique médicale exposée dans cet article. Car il aborde la séméiotique dans la Lettre sur les aveugles à partir d'une anatomie générale de la perception, présentée par ailleurs comme une «anatomie métaphysique ». Dans une perspective relativement proche de celle relevée chez Leibniz, Diderot distingue un ordre naturel, un « ordre d'institution » et un « ordre scientifique » des idées ou «vues de l'esprit » ${ }^{44}$. Or l'ordre des vues de l'esprit n'est pas scientifique en lui-même, il le devient s'il suit la méthode d'invention conçue par Diderot. Rappelons que celui-ci critique l'Encyclopédie de Chambers pour son absence d'invention: Chambers, " $n$ 'inventant rien, s'en tenant rigourcuscment aux choses connues », adopte un ordre d'une régularité exemplaire, mais les différents articles qui le composent « sont vides ». Tout se passe comme si l'ordre encyclopédique traduisait un certain art d'inventer et reflétait les «opérations de l'esprit » les plus inconscientes. De même que pour Leibniz, l'ordre scientifique obéit à la «méthode d'invention ${ }^{45}$. Il représente une sorte d'idéal encyclopédique selon lequel l'ordre des connaissances reviendrait à un enchaînement de conjectures. Il faudrait pour cela, imagine Diderot, qu'il y ait « en quelque endroit du monde un livre magique qu'on pût toujours consulter, et où toutes les pensées des hommes allassent se graver au moment où elles existent dans l'entendement $\gg{ }^{46}$. L'Encyclopédie idéale serait ce « livre magique ». Diderot estime que depuis «la collection générale de toutes les causes jusqu'à l'être solitaire, tout a son signe ». Autrement dit, les phénomènes représentent «autant de vérités partielles ». Tandis que l'invention, conçue comme combinatoire, traduit leur enchaînement, de telle sorte que l'on passe de la sensation simple à l'idée abstraite et d'une collection de signes à un ordre général. Il en résulte une abréviation similaire à celle envisagée par Leibniz. Car loin de multiplier les points de vue ${ }^{47}$, l'encyclopédisme rejoint, par

44. Diderot, Lettre sur les sourds et muets, DPV IV, pp. 140 et 137.

45. Diderot, Encyclopédie, article ENCYCLOPÉDIE, DPV VII, pp. 215-216, pp. 193 et 197 et p. 219 . Dans une perspective proche de Condillac, Diderot affirme que la langue rend compte des «opérations de l'esprit ».

46. Diderot, Encyclopédie, article ÉCLECTISME, DPV VII, 47.

47. Diderot, Encyclopédie, article ENCYCLOPÉDIE, DPV VII, pp. 190) et 192 et pp. 208-209. « Faut-il qu'un dictionnaire contienne autant de fois un mot qu'il y a de différences dans les vues de l'esprit? l'ouvrage serait infini, et ce sera nécessairement un chaos de répétitions ». 
sa méthode, les mathématiques : «Le géomètre renvoie d'un théorème ou d'un problème à un autre et l'encyclopédiste d'un article à un autre ». Cette intelligence des renvois constitue d'ailleurs la «partie de l'ordre encyclopédique la plus importante », car elle obéit au principe d'analogie qui guide l'invention. Celleci, définie comme un «esprit de combinaison », multiplie le nombre de renvois, renvois qui, « en rapprochant dans les sciences certains rapports, dans les substances naturelles des qualités analogues, dans les arts des manœuvres semblables, conduiraient ou à de nouvelles vérités spéculatives, (...) ou à l'invention de nouveaux arts ${ }^{48}$. L'Encyclopédie s'oppose ainsi à l'académisme, où les connaissances sont dispersées dans les différents auteurs et comparées à des phénomènes isolés qui faute de liaison ne produisent ni sensation ni idée nouvelle ${ }^{49}$.

La conception de l'ordre encyclopédique des connaissances s'avère quasiment analogue chez Leibniz et Diderot ; ce qui nous amène à repenser la distinction mise en relief par $\mathrm{Y}$. Belaval entre combinatoire intellectuelle et combinatoire sensible, ou encore à redéfinir le rapport entre la séméiotique et l'«anatomie ». Car Diderot compare le cerveau, organe de la mémoire, à un véritable « livre vivant » résistant à la lecture. Une telle métaphore illustre l'idée d'une anatomie confrontée à la complexité indéchiffrable de l'organisation. "Point de signes si disparates qui ne confinent. Point d'idées si bizarres qui ne se touchent $\gg{ }^{50}$ dans l'organe qu'est le cerveau. «Les caractères de ce livre vivant ne vous sont pas encore connus ; peut-être ne vous le seront-ils jamais ; mais les dépositions des cinq témoins n'y sont pas moins consignées, combinées, comparées, confrontées » ${ }^{51}$, écrit Diderot qui trace ici un rapport explicite entre séméiotique et anatomie.

Ce rapport s'explique chez Diderot par un ensemble d'analogies, sensibles certes mais aussi arbitraires et conventionnelles. Il s'explique dans le système de Leibniz par l'hypothèse de l'harmonie préétablie. Car s'il existe un ordre naturel, celui-ci résulte de l'harmonie préétablie entre le corps et l'âme ; c'est

48. IBID., DPV VII, pp. 227,221 et 223. La « fréquence des renvois » reflète «l'universalité des connaissances ».

49. IBID., DPV VII, 179. Les mémoires académiques renferment « une infinité de matériaux excellents dispersés dans un grand nombre d'ouvrages, où ils restent sans produire aucune sensation utile, comme des charbons épars qui ne formeront jamais de brasier ».

50. Diderot, Salon de 1767, DPV XVI, 219.

51. Diderot, Réfutation d'Helvétius, VER. T. I, 825. 
l'ordre des petites perceptions. Celles-ci sont à l'origine des notions confuses acquises par la perception sensible. Mais ces notions sont tellement composées que nous nous figurons « quelque réalité nouvelle » ${ }^{52}$ plutôt que d'en faire l'analyse : « nous substituons alors aux choses des signes $\gg{ }^{53}$. Dès lors, la connaissance peut être dite «symbolique », dans la mesure où la part « occulte » ${ }^{54} \mathrm{du}$ sensible est remplacée par des signes. La connaissance symbolique est donc la seule connaissance que nous ayons du sensible. Elle est opératoire et procède à une composition naturelle de notions dont la réalité est continuellement mise à l'épreuve de façon expérimentale. Nous nous contentons le plus souvent d' « apprendre de l'expérience la réalité de certaines notions et de nous servir ensuite de ces notions pour en composer d'autres à l'exemple de la nature » ${ }^{55}$. La combinatoire serait néanmoins impossible si certaines notions abstraites, qui sont à la fois «sujettes à l'imagination » et «objets des sciences mathématiques », comme notamment la notion de nombre, ne se mêlaient à notre insu aux différentes qualités sensibles. C'est pourquoi Leibniz parle d'une «Arithmétique occulte», selon laquelle l'âme fait « une infinité (...) [de] petites opérations très justes, quoiqu'elles ne soient point volontaires ni connues $\gg{ }^{56}$. Cette «Arithmétique naturelle » ou ce calcul est pour Leibniz «plus important que ceux de l'Arithmétique et de la Géométrie, et (...) dépend de l'Analyse des idées », ou encore de leur « anatomie ».

Car l'anatomie rejoint ici la séméiotique, qui relève d'une imagination corporelle et machinale. Les pensées les plus abstraites trouvent leur jeu dans le corps, ceci au moyen de signes ou de «caractères qui les représentent à l'imagination » 57 . Le corps et les organes, y compris le cerveau, perçoivent la réalité selon

52. Leibniz, Méditations sur la connaissance, la vérité et les idées, Guvres de G. W. Leibniz, éd. Prenant, Aubier Montaigne, (1972), T. I, p. 156.

53. Leibniz, Méditations sur la connaissance, la vérité et les idées, Leibniz, Opuscules philosophiques choisis, éd., Schrecker, Vrin, (2 éd., 2001), p. 17.

54. Leibniz, Lettre touchant ce qui est indépendant des sens et de la matière, GP VII, 499. Les « qualités occultes » sont les qualités que nous percevons mais «ne voyons pas ».

55. Leibniz, Méditations sur la connaissance, la vérité et les idées, éd. Schrecker, p. 25.

56. Leibniz, Extrait du Dictionnaire de Bayle, article Rorarius, p. 2599 sqq. de l'Édition de l'an 1702 avec mes remarques. GP IV. 551.

57. Leibniz, Réponse aux réflexions contenues dans la seconde Édition du Dictionnaire Critique de M. Bayle, article Rorarius, sur le Système de l'Harmonie préétablie, GP IV. pp. 556, 571, 562 et 559. 
une «tablature » de signes, tandis que l'âme en a la mémoire, ou, précise Leibniz, quelque chose d'équivalent. L'anatomie s'inspire, semble-t-il, de la séméiotique, ce qui donne plus de force à l'hypothèse de l'harmonie préétablie. Le corps, véritable automate naturel, est comparable aux automates artificiels que sont les « montres sonnantes » et les « réveil-matin »: «bien loin d'attendre des signes, ils nous en donnent ${ }^{58}$, s'exclame Leibniz. Il est dès lors possible de concevoir le rapport entre les deux substances selon un modèle qui s'oppose à la thèse de l'influence et s'inspire de la séméiotique. Car le rapport entre les substances est analogue à celui que l'on observe entre une suite quelconque de signes et la suite des pensées qui y correspond. La connaissance symbolique procède par signes et l'on comprend pourquoi Leibniz peut affirmer que la substance est à «elle-même la source de ses phénomènes ${ }^{59}$.

Selon Diderot, dont il reste à étudier la théorie de la sensation dans son rapport à la séméiotique, l'analogie entre le réel et l'ordre des sensations n'est ni préétablie ni véritablement naturelle, comme dans la théorie leibnizienne des petites perceptions. La sensation repose sur un certain nombre d'analogies, qui sont en grande partie d'institution et que l'expérience ne cesse de mettre à l'épreuve : " les sensations n'ayant rien qui ressemble essentiellement aux objets, c'est à l'expérience à nous instruire sur des analogies qui semblent être de pure institution $\gg{ }^{60}$. C'est dans cette perspective que Diderot développe ce qu'il appelle un «anatomie métaphysique " ${ }^{61}$, anatomie selon laquelle la connaissance acquise par les sens résulte de l'imagination spécifique de chacun d'eux; ceci différemment de Leibniz pour qui seules les qualités communes aux divers sens sont sujettes à imagination. Enfin, la connaissance passe par des signes. « Les

58. Leibniz, Extrait, GP IV, 541. Leibniz écrit que l'âme «trouve par ellemême et même sans peine et sans application, et sans le chercher ce que le cerveau et ses organes trouvent par l'aide du livre », IBID., GP IV, 549.

59. Leibniz, Réponse, GP IV, 559.

60. Diderot, Lettre sur les aveugles, DPV IV, 62. Sur la théorie de la connaissance de la Lettre sur les aveugles, voir G. Stenger, « La théorie de la connaissance dans la Lettre sur les aveugles », Recherches sur Diderot et sur l'Encyclopédie, numéro 26, avril 1999, pp. 99-111.

61. Diderot, Lettre sur les sourds et muets, DPV IV, 140. L'expression désigne les analyses menées dans la Lettre sur les aveugles et complétées dans la Lettre à Mademoiselle de Chaux. Diderot évoque déjà l'idée d'une anatomie de l'âme, capable de «sonder ses blessures et parcourir ses replis », dans une note de sa traduction de l'Essai sur le mérite et la vertu, DPV I, 365. 
connaissances ont trois portes pour entrer dans notre âme ; et nous en tenons une barricadée, par le défaut de signes ». Diderot s'intéresse par conséquent à l'imagination tactile ${ }^{62}$ développée par les aveugles, ainsi qu'à l'«arithmétique palpable »63 qui combine les signes acquis par le toucher. Il retrouve la « table (...) des qualités sensibles, et des notions abstraites ; communes et particulières à chacun des sens " ${ }^{64}$, évoquée par Leibniz. Ces notions abstraites, la plus abstraite étant celle des nombres, sont d'abord conservées par une mémoire organique qui se limite à un simple dénombrement de sensations ${ }^{65}$. La connaissance la plus abstraite que nous ayons du sensible est aussi la plus élémentaire mais « l'unité pure est simple est un symbole trop vague et trop général pour nous »: «Nos sens nous ramènent à des signes plus analogues à l'étendue de notre esprit et à la conformation de nos organes ». Diderot évoque, selon une terminologie proche de celle de Leibniz, l'image d'une " tablature » ${ }^{66}$ propre à chacun des sens. Les signes mettent en jeu l'imagination spécifique de chaque sens. Selon cette séméiotique pré-linguistique, c'est-à-dire antérieure à la fixation des signes dans la langue, il y aurait autant de variété de signes que de sens : ce sont les caractères pour les yeux, les sons articulés pour l'oreille et Diderot souligne l'absence d'une langue tactile pour le toucher.

62. Diderot, Lettre sur les aveugles, DPV IV, p. 34 et pp. 30-31. « Nous ne distinguons la présence des êtres hors de nous, de leur représentation dans notre imagination, que par la force et la faiblesse de l'impression : pareillement. l'aveugle-né ne discerne la sensation d'avec la présence réelle d'un objet à l'extrémité de son doigt, que par la force ou la faiblesse de la sensation même ». Diderot fait ici un parallèle entre l'imagination visuelle et l'imagination tactile, en faisant appel aux notions d' « intensité » et de « rémission », notions mathématiques communes aux différents sens. Ces notions ont déjà été relevées par Leibniz dans le cadre de la théorie de la perception, Lettre touchant ce qui est indépendant des sens et de la matière, GP VI, 501.

63. Diderot, Lettre sur les aveugles, DPV IV, 34. L'expression est empruntée au titre de l'ouvrage rédigé par J. Colson, successeur de Saunderson, inventeur de la méthode, à la chaire de mathématiques de Cambridge. John Colson, Dr Saunderson's Palpable Aritmetic, in The Elements of Algebra. Cambridge University Press, 1740, pp. XX-XXVI.

64. Diderot, Lettre à Mademoiselle de Chaux, DPV IV, 196.

65. Diderot, Encyclopédie, article ENCYCLOPÉDIE, DPV VII, 194. Diderot parle de la «mémoire ou conscience d'une, de deux. trois, quatre, etc. perceptions différentes; ou celle de la même perception une, deux, trois, quatre fois réitérée : et par conséquent la notion des nombres, un, deux, trois, quatre, etc. ». Cette mémoire est dite «passive» dans le Prospectus de l'Encyclopédie, DPV V, 105.

66. Diderot, IBID., DPV VII, 200. L'image de la «tablature » s'explique par la comparaison des organes des sens avec des instruments de musique. 
Selon cette « anatomie métaphysique », la connaissance résulte d'une combinatoire de signes dont la sensation sert à multiplier le nombre. Plus précisément encore, l'analogie entre les notions abstraites et la sensation se vérifie par une «suite de combinaisons fines et profondes $\gg{ }^{67}$. Ici encore, il s'avère difficile d'affirmer qu'il s'agit d'une combinatoire strictement sensible ou strictement intellectuelle. C'est en fait une " géométrie expérimentale » dont les éléments sont plus composés que ceux de la "géométrie intellectuelle ${ }^{68}$, selon une définition qui rejoint la distinction leibnizienne de la théorie et de la pratique. Diderot pense ici aux différents arts et métiers. Les notions abstraites sont acquises par l'exercice des sens et une mémoire organique selon laquelle l' " on combine les idées et les signes en une infinité de manières différentes ${ }^{69}$. C'est ainsi que nous nous donnons des signes et développons des «prénotions » que nous multiplions par « un usage assidu des sens ${ }^{70} ;$ «prénotions » sans lesquelles, estime Diderot, « rien de particulier n'est présent à l'esprit ». Celles-ci, de même que les autres signes pré-linguistiques, sont conservées par une mémoire dite " artificielle " ${ }^{7}$. Ainsi, toute sensation ou jugement se fait sur un «fond» composé de signes acquis par l'usage des sens. La thèse est illustrée par la Lettre sur les aveugles, où Diderot envisage le cas d'un métaphysicien qui recouvrerait la vue. Celui-ci, contrairement aux autres personnes ayant subi la même opération, apercevrait "distinctement les objets, comme s'il les avaient vus toute sa vie $"{ }^{72}$. Car il aurait plus que quiconque l'habitude de combiner des idées et des signes. Cette hypothèse constitue une réponse originale au problème de Molyneux. Elle est à rapprocher de l'article LOCKE de l'Encyclo-

67. Diderot, Lettre sur les aveugles, DPV IV, 25.

68. Diderot, Encyclopédie, article ART, DPV V, 502-503.

69. Diderot, Encyclopédie, article ENCYCLOPÉDIE, DPV VII, 206.

70. Diderot, Encyclopédie, article ÉPICURISME, DPV VI, 269. « Loin de pouvoir travailler à la recherche de la vérité, on n'est pas même en état de se faire des signes. Multipliez donc les prénotions par un usage assidu de vos sens ».

71. Diderot, Encyclopédie, Prospectus, DPV V, 110. Diderot distinguc unc «mémoire naturelle », qui est « une affection des organes», et une mémoire « artificielle » qui « consiste dans la prénotion et dans l'emblème ». L'intelligence de «l'emblème poétique » et les problèmes liés à l'actualisation des sensations et des prénotions par la mémoire naturelle sont évoqués dans la Lettre sur les Sourds et Muets, DPV IV, p. 163, et pp. 169-176, ainsi que dans le Salon de 1767, DPV XVI, 217. Voir James Doolittle, "Hieroglyph and emblem in Diderot's Lettre sur les sourds et muets », Diderot's Studies II, Syracuse University Press, pp. 148-167.

72. Diderot, Lettre sur les aveugles, DPV IV, p. 67 et pp. 68-70. 
pédie, dans lequel Diderot explique que l'on combine d'autant mieux les signes, les idées et les sensations, que l'on en multiplie le nombre par l'observation et l'expérience. Il note en effet que Locke est passé « de l'étude du cartésianisme à celle de la médecine », ce qui l'a amené à considérer «l'homme sous une infinité de points de vue intéressants ». Diderot conclut qu'il « n'appartient qu'à celui qui a pratiqué la médecine pendant longtemps d'écrire de la métaphysique ; c'est le seul qui a vu les phénomènes ${ }^{73}$. Autrement dit, le métaphysicien se doit d'être séméioticien, au sens médical du terme, tout se passant comme si pour le matérialisme diderotien la séméiotique pouvait tenir lieu de métaphysique.

La séméiotique de Diderot rejoint ainsi les termes de l'article anonyme SIGNE de médecine séméiotique de l'Encyclopédie, termes selon lesquels « le phénomène ou symptôme peut devenir un signe lorsqu'on cesse de le considérer abstractivement ${ }^{74}$, c'est-à-dire isolément. Si le signe a valeur de prénotion et anticipe sur l'enchaînement des idées, il a aussi, selon la séméiotique médicale, valeur de "prognostic ${ }^{75}$ et anticipe sur l'ordre des symptômes et des phénomènes. Tant et si bien que la perception, parce qu'elle est aussi une combinatoire de notions abstraites et de signes, anticipe sur le réel. On retrouve au travers de la séméiotique et de l'《anatomie métaphysique » de Diderot l'idée d'inquiétude comme sensibilité à l'avenir, un paradoxe que Leibniz soulignait déjà dans les Nouveaux essais ${ }^{76}$. Or la sensibilité à l'avenir ne saurait se réduire à une combinatoire intellectuelle ou à un calcul des possibles.

Claire Fauvergue Université de Nagoya

73. Diderot, Encyclopédie, article LOCKE PHILOSOPHIE DE, DPV VII, 710.

74. Encyclopédie, article sicine, (Médecine séméiotique), non signé, Enc: XV, $188 \mathrm{~b}$

75. Ménuret de Chambaud, Encyclopédie, article PROGNOSTIC, Enc., XIII, 429 $\mathrm{a}-\mathrm{b}$.

76. Leibniz, Nouveaux essais, GP V, 190. L'avenir nous touche, bien que nous n`en ayons aucun « signe naturel». 